toid disease. Proceedings of a symposium at Spåtind, Norway. Oslo: Fabritius and Sønner, 1976: 152-60.

5 Stanworth D R. The role of IgA in the pathogenesis of rheumatoid arthritis. In: Goodacre J,
Carson Dick W, eds. Immunopathogenesis of Carson Dick W, eds. Immunopathogenesis of
rheumatoid arthritis. Lancaster: MTP Press, 1988: $122-42$

6 Dawes P T, Jackson R, Shadforth M F, Lewin I V, Stanworth D R. The relationship between the complex of immunoglobulin $\mathrm{A}$ and alpa-1antitrypsin, its constituent components and the acute phase response as measured by $\mathrm{C}$-reactive protein in rheumatoid arthritis treated with gold or D-penicillamine. Br $\mathcal{F}$ R heumatol 1987; 26: 351-3.

7 Davis M J, Dawes P T, Fowler P D, Shadforth $M$ F, Lewin I V, Stanworth D R. The association and predictive value of the complex immunoglobulin A- $\alpha$ l-antitrypsin in the development of erosions in early rheumatoid arthritis. Scand $\mathcal{F}$ Rheumatol 1991; 20: 23-7.

AUThORs' REPLY We were interested to read Dr Stanworth's letter, in which he recalled his thoughts in the 1970s about the mechanism of action of D-penicillamine and his more recen experiments on the $\operatorname{IgA-} \alpha_{1}$ antitrypsin complex. Dr Israeli Jaffe, very reasonably, had suggested, following his studies of intraarticular injection of penicillamine, that this drug degraded IgM rheumatoid factor (IgM RF) by sulphydryl reduction of the IgM RF molecule. We were uncertain that this was the mechanism because we reasoned that the therapeutic effect should occur more rapidly than the several months required if a simple chemical reduction of IgM RF took place. It seemed to us that it was more likely that the delayed onset of clinical improvement was associated with a gradual change in the cellular immune response in the synovial membrane due, perhaps, to a progressive inactivation of a critical cell population playing a part in this response. We reasoned that this should be reflected in a gradual fall in the titre of rheumatoid factor. The fall in titre might not necessarily be the reason for the joint improvement but, rather, an associated change.

It was for this reason that we decided to measure the titres of IgM RF over time in patients receiving penicillamine. It turned out that the titre fell gradually over a period of months. As pointed out by Dr Stanworth, in subsequent experiments carried out in collaboration with Dr Jaffe, our laboratory showed that there was no correlation between RF titre and clinical improvement in patients treated with penicillamine. ' Similar results have been reported by several other groups. In later experiments carried out in Dallas Olsen et al noted a correlation between spontaneous synthesis of IgM RF by peripheral blood mononuclear cells and disease activity in patients treated with penicillamine. ${ }^{2}$ This result suggested that penicillamine suppressed the release of cells producing RF into the circulation possibly from the inflamed synovium itself. Thus this observation was also compatible with a cellular mechanism. Lipsky and Ziff have, in fact, shown that penicillamine inhibits helper $\mathbf{T}$ cell function in the presence of copper. $^{3}$

We are aware of the observations that the complex of immunoglobulin $A$ and $\alpha_{1}$ antitrypsin is reduced by gold and $\mathrm{D}$-penicillamine Recent data suggesting that the levels of serum IgA- $\alpha_{1}$ antitrypsin complex might be a predic tive indicator of erosions in early rheumatoid arthritis are of interest. Undoubtedly, further studies will tell us if this is the only predictor. If indeed $D$-penicillamine can reduce this complex, then we would expect erosions to heal, or perhaps not occur, if the complex level is reduced by treatment. We are no aware that studies to date have provided overwhelming evidence for such a role for Dpenicillamine.

Fortunately, many treatments introduced into medicine for the wrong reason have still been effective and have stimulated subsequent excellent research.

EVELYN V HESS Cincinnati
Ohio, USA MORRIS ZIFF Dallas,
Texas, USA

Correspondence to: Professor E V Hess, Department of Internal Medicine, Division of Immunology, Mail Location 563, 231 Bethesda Avenue (Room 7562), Cincinnati, Ohio 4267-0563, USA.

I Wernick R, Merryman P, Jaffe I, Ziff M. IgC and $\mathrm{R} M$ rheumatoid factors in and IgM rheumatoid factors in rheum

2 Olsen N, Ziff M, Jasin H. Spontaneous synthesis of IgM rheumatoid factor by blood mononuclear cells from patients with rheumatoid arthritis: effect of treatment with gold salts or D-penicillamine. F R heumatol 1984; 11: 17-21.

3 Lipsky P E, Ziff M. Inhibition of human helper copper sulfate. $\mathcal{F}$ Clin Invest 1980; 65: 1069-76.

\section{von Willebrand factor antigen in giant cell arteritis}

Sir: We were surprised at Dr Nordborg and colleagues somewhat negative appraisal of their findings in following von Willebrand factor (vWF) and plasminogen activator inhibitor levels in a large cohort of patients with giant cell arteritis. ${ }^{1}$ Although showing that vWF levels are not useful in monitoring clinical relapse, they did establish that vWF levels gradually fall to control values after 18-24 months, which corresponds with the natural history of the disease in most patients. This is an important observation as several studies have concluded that VWF is raised but not useful in assessing clinical activity and have noted a persistent increase in $\mathrm{VWF}$ after successful corticosteroid treatment. ${ }^{2-4}$

The logical conclusion from Dr Nordborg's data is that vWF levels reflect not only vascular injury but the activity of the fundamental disease process in giant cell arteritis. It also supports the argument that steroid treatment, although mitigating vascular injury progressing to thrombotic/embolic occlusion, has little effect on the underlying disease. In this study 'nine of 63 patients still receiving corticosteroids had a . . . vascular occlusive episode'. Moreover, the use of vWF as a marker obscures its pathogenic potential via its main function, the initiation of platelet adhesion. von Willebrand factor release at the site of vascular injury is the vital first step in the thrombotic cascade, which even when it does not lead to occlusion will perpetuate vascular injury by the release of platele derived vasoactive substances and growth factors. There is a strong correlation between vWF levels and such growth factors after myocardial infarction. ${ }^{5}$ Of further pathogenetic relevance is the close relation between vWF release and expression of the endothelial cell polymorph adhesion molecule GMP-140, which occurs because both are stored in Weibel-Palade bodies.

Thus the principal implication is that in relapsing cases or those unresponsive to steroids there should be a low threshold for using agents which could modulate or modify either vascular injury or the disease process and that $\mathrm{VWF}$ responses might provide clues to the most effective drugs and their optimal usage. von Willebrand factor responses may also help to identify new immunomodulators. Indeed, given that vWF release is $\mathrm{Ca}^{2}$ dependent, it is tempting to speculate about the therapeutic effect of calcium channel blockers in giant cell arteritis and other vasculitides.

\section{A J FARREL D R BLAKE matology Unit Notingham NG5 IPB}

1 Nordborg E, Andersson R, Tengborn L, Eden S, Bengtsson B A. von Willebrand factor antigen and plasminogen activator inhibitor in giant cell arteritis. Ann Rheum Dis 1991; 50: 316-20.

Cimpi 1 . Bem M L, Marotta G, Puccetti L, et al. Behaviour of von Willebrand factor antigen in Rheumatol 1988; 17: 491-5.

3 Persellin S T, Daniels T M Rings I J et al. Factor VIII-von Willebrand factor in giant cel arteritis and polymyalgia rheumatica. Mayo Clin Proc 1985; 60: 457-62.

4 Federici A B, Fox R I, Espinoza L R, Zimmerma $T S$. Elevation of von Willebrand factor is independent of ESR and persists after glucocorticoid treatment in giant cell arteritis. Arthritis Rheum 1984; 27: 1046-9.

5 Nilsson J, Elgue G. Wallin M, et al. Correlation between plasma levels of growth factors and von Willebrand factor. Thromb Res 1989; 54 : 125-32.

\section{Renal cell carcinoma with acute monarthritis}

Sir: We read with interest the report of two cases of renal cell carcinoma presenting with acute monarthritis. ' We would like to report a similar case.

A 55 year old woman presented in June 1988 with a painful left knee after a walking holiday. Although arthroscopy was carried ou on her knee in May 1989, showing norma joint surfaces and an inflamed synovium which bled easily, no biopsy sample or radiograph was taken. When seen by us in Novembe there was considerable tender, warm swelling. Radiography showed a large osteolytic lesion in the lower left femur with a pathological fracture and a surrounding soft tissue mas (figure). Histology showed an invading clea cell tumour. Further investigations to find th primary tumour disclosed a second metastatic deposit in the right shoulder and two small metastatic nodules in the lung. Abdomina computed tomography showed a large mass arising from the right kidney. Treatment proceeded to nephrectomy, which confirmed poorly differentiated clear cell tumour with invasion into the capsule, ureter, and renal vein. She received radiotherapy to the femur, and the left knee was reconstructed successfully. Interferon was given, but the patien died in early August 1990.

Metastatic bone disease is found in $49 \%$ of patients with renal cell carcinoma. ${ }^{2}$ Interestingly, it is reported ${ }^{3}$ that bony metastases are usually ipsilateral to the primary tumour and rarely affect peripheral bones. This is thought to be because the paravertebral veins form a rich plexus extending from the skull to the knees and elbows by way of its connections with the vasa vasorum. Frequent connections between the renal vein and the paravertebral plexus occur directly on the left but indirectly on the right. Of the $\mathbf{4 0}$ patients discussed with bony metastases, 10 had axial involvement alone (three right sided primary tumours, seven left sided) and a further 15 also had 